\title{
Endovascular therapy of low- and intermediate-grade intracranial lateral dural arteriovenous fistulas: a detailed analysis of primary success rates, complication rates, and long-term follow-up of different technical approaches
}

\author{
Lorenz Ertl, MD, MSc, ${ }^{1}$ Hartmut Brückmann, MD, ${ }^{1}$ Mathias Kunz, MD, ${ }^{2}$ \\ Alexander Crispin, MD, MPH, ${ }^{3}$ and Gunther Fesl, MD, MHBA ${ }^{1}$ \\ 'Department of Neuroradiology, Institute of Clinical Radiology, ${ }^{2}$ Department of Neurosurgery, and ${ }^{3}$ Institute for Medical \\ Informatics, Biometry, and Epidemiology (IBE), University of Munich, Germany
}

\begin{abstract}
OBJECTIVE Sinus-preserving (SP) embolization techniques augment endovascular treatment options for intracranial lateral dural arteriovenous fistulas (DAVFs). The authors aimed to perform a retrospective comparison of their primary success rates, complication rates, and long-term follow-up with those of sinus-occluding (SO) treatment variants in the collective of low- and intermediate-grade lateral DAVFs (Cognard Types I-IIb).

METHODS Clinical symptoms, complication rates, and Cognard grading prior to and after endovascular DAVF treatment using different technical approaches was retrospectively analyzed in 36 patients with lateral DAVF Cognard Types I-Ilb. The long-term success rate was determined by a standardized questionnaire.

RESULTS The SO approaches offered a higher rate of definitive fistula occlusion ( $93 \%$ SO vs $71 \%$ SP) but were accompanied by a significantly higher complication rate ( $33 \%$ or $20 \%$ SO vs $0 \%$ SP). The patients interviewed reported very high satisfaction with their health in long-term follow-up in both groups.

CONCLUSIONS A higher rate of definitive fistula occlusion in the SO group was attained at the price of a significantly higher complication rate. The SP approaches offered a good primary success rate in combination with a very low complication rate. Despite some limitations of the data (e.g., a small sample size) the authors thus recommend an SP variant as the primary therapeutic option for the endovascular treatment of low- and intermediate-grade DAVFs. The SO approaches should be restricted to cases in which SP treatment does not achieve a downgrading to no worse than Cognard Type lla.
\end{abstract}

http://thejns.org/doi/abs/10.3171/2016.2.JNS152081

KEY WORDS intracranial fistula; endovascular treatment; therapy assessment; interventional neurosurgery

I NTRACRANIAL dural arteriovenous fistulas (DAVFs) are pathological shunts between dural arteries and dural venous sinuses, meningeal veins, or cortical veins. DAVFs account for $10 \%-15 \%$ of intracranial arteriovenous malformations. ${ }^{9}$ The venous drainage pattern of a DAVF determines its severity and classification according to Borden et al. ${ }^{1}$ and Cognard et al. ${ }^{3}$ Both of these systems associate cortical venous drainage with increased risk of intracranial hemorrhage and neurological deficits. , $^{2,6,7,13}$, 15,16

During the past 2 decades, embolization has become the first-line treatment for DAVFs. In particular, the inven- tion of highly compliant, inflatable occlusion balloons and the Onyx liquid embolic agent (ev3 Neurovascular) opened a new horizon in the endovascular treatment of DAVFs. By temporarily inflating a large-lumen compliant balloon in the diseased sinus compartment during the injection of Onyx into an arterial feeder, this combined sinus-preserving embolization (CSPE) approach is supposed to preserve the natural venous drainage pattern and allow a better penetration of the fistula network by the liquid embolic agent.

Transvenous embolization (TVE) is performed by retrograde catheterization of the involved dural sinus or cortical vein, followed by deposition of coils and/or liquid

ABBREVIATIONS CCA = common carotid artery; CCF = carotid cavernous fistula; CSOE = combined sinus-occluding embolization; CSPE = combined sinus-preserving embolization; DAVF = dural arteriovenous fistula; DSA = digital subtraction angiography; ECA = external carotid artery; SO = sinus-occluding; SP = sinus-preserving; TAE = transarterial embolization; TVE = transvenous embolization

SUBMITTED September 4, 2015. ACCEPTED February 3, 2016.

INCLUDE WHEN CITING Published online April 29, 2016; DOI: 10.3171/2016.2.JNS152081. 
embolic agents adjacent to the shunt. ${ }^{5,12}$ The occlusion of a dural sinus, however, carries a risk of venous infarction or hemorrhage, which significantly limits this therapeutic approach. The challenge is to obtain a controlled gradual DAVF obliteration without any diversion of arterialized blood to the cortical veins. ${ }^{10}$

Transarterial embolization (TAE) requires superselective distal catheterization of arterial feeders. Ideally, the microcatheter tip should be "wedged" in the feeding artery. An embolic agent should then penetrate the fistulous connection and proximal aspect of the venous receptacle..$^{14}$ Available embolic agents include particles, coils, ethanol, N-butyl cyanoacrylate glue, and Onyx..$^{5,11}$ Caution should be exercised to avoid accidental embolization of the distal venous system, which may result in progressive venous occlusion, exacerbation of venous hypertension, and/or venous infarction..$^{8,14}$

The combination of both techniques by occlusion of the draining sinus compartment from the venous side and embolization of the arterial feeders (combined sinusoccluding embolization [CSOE]) was supposed to offer a higher rate of definite occlusion. However, potential complications resulting from a compromise of the natural intracranial venous drainage pathways remain the same as with TVE.

Sinus-preserving (SP) treatment variants today are favored in many centers, but there has not yet been a structured evaluation of their benefit. In this article we aim to conduct a retrospective comparison of the different approaches of endovascular treatment for DAVFs performed in our department in the past 2 decades in the collective of low- and intermediate-grade fistulas (Cognard Types I$\mathrm{IIb})$.

\section{Methods \\ Study Population}

We retrospectively reviewed patient records from January 2003 to June 2015 to identify a subset of patients who received endovascular treatment of an intracranial DAVF at the neuroradiology department of our institution. From these records we excluded all carotid cavernous fistulas $(\mathrm{CCF})$ and any DAVF primarily draining into the frontobasal/ethmoidal sinus or superior sagittal sinus. The remaining DAVFs, primarily involving the sigmoid and/ or the transverse sinus, were defined to be of a lateral type and graded according to the Cognard classification scheme (Table 1). We included all lateral DAVFs with a grade of Cognard Types I-IIb in our analysis (Table 2).

\section{Endovascular Intervention}

All endovascular interventions were performed by the same trained neuroradiologists on a biplane neuroangiography suite (Neurostar Top or Axiom Artis Zee Biplane, both Siemens AG). Materials used during intervention are listed in Table 3. We used Histoacryl (B. Braun), Glubran (GEM), Onyx 18 liquid embolic agent, coils (different sizes, lengths, and manufacturers), and a venous remodeling balloon (Copernic $8 \times 80 \mathrm{RC}$, BALT Extrusion SAS) in different combinations (Table 3 ).

\section{Evaluation Criteria}

All patient records were reviewed to determine the clinical symptoms and the fistula grading according to Cognard et al. at the time of initial presentation. The imaging material was reanalyzed in detail by 2 experienced neuroradiologists (L.E. and G.F., with 7 and 15 years of experience, respectively). The date of treatment was noted, and the interval between initial diagnosis and treatment was calculated. The methodological treatment approach (TVE, TAE, CSOE, or CSPE), intervention material, and any complications during the treatment were noted. We defined a complication as any technical problem with the material used or any medical problem occurring during the intervention.

The date of the last imaging follow-up was noted, and the interval from treatment to the end of imaging followup was calculated. The date of the last imaging follow-up marked the evaluation point of the primary therapeutic success rate. To determine the primary success rate, each fistula was graded according to the Cognard classification system on the last imaging follow-up. We analyzed any digital subtraction angiography (DSA) and MRI available during follow-up, but still considered DSA to be the gold standard.

For statistical purposes, we additionally established a scoring system for the comparison of the primary therapeutic success rate: if imaging at last follow-up was negative for a remaining arteriovenous shunt, the therapeutic success rate was rated as 3 points. Any downgrading in the Cognard scale with persistent arteriovenous shunt scored 2 points. If treatment led to a reduction of shunt volume and/or size of the fistula network, but did not achieve a downgraded Cognard type, the treatment success rate was rated as 1 point. If no treatment effect was noticeable, the success rate was scored with 0 points (Table 4 ).

To determine the long-term success rate a standardized questionnaire was sent to all patients inquiring about remaining clinical symptoms and their subjective perception of the long-term treatment by subjectively comparing their current state to that before the intervention.

\section{Data Collection and Statistical Analysis}

All data were collected in a custom-designed database using standard software (Access 2010, Microsoft). All data of every patient were reviewed, and a scoring sheet in the database was completed.

For the statistical comparison, the total collective of patients was divided into 2 groups defined by the functionalanatomical state achieved at the definitive end of treatment $([\mathrm{SO}$ embolization $=\mathrm{TVE}+\mathrm{CSOE}]$ vs $[\mathrm{SP}$ treatment $=$ TAE + CSPE]). In the case of several sessions with different approaches, the functional anatomical result of the last intervention defined the group assignment.

All statistical analysis was performed using SPSS (version 22.0.0.0, IBM Corp.). It included descriptive statistics for the demographic data and the timeline of the treatment process and Fisher's exact tests for group comparisons regarding complication rates and primary therapeutic success rate. The ethics committee of our institution approved this study. 
TABLE 1. Cognard classification scheme and characteristics

\begin{tabular}{|c|c|c|c|c|c|c|}
\hline I & Ila & Ilb & Ila+b & III & IV & V \\
\hline $\begin{array}{l}\text { Confined to } \\
\text { sinus }\end{array}$ & Confined to sinus & $\begin{array}{l}\text { Drains into si- } \\
\text { nus w/ reflux } \\
\text { into cortical } \\
\text { veins }\end{array}$ & $\begin{array}{l}\text { Drains into sinus } \\
\text { w/ reflux into } \\
\text { cortical veins }\end{array}$ & $\begin{array}{l}\text { Drains di- } \\
\text { rectly into } \\
\text { cortical } \\
\text { veins }\end{array}$ & $\begin{array}{l}\text { Drains directly } \\
\text { into cortical } \\
\text { veins }\end{array}$ & $\begin{array}{l}\text { Spinal perimedullary } \\
\text { venous drainage }\end{array}$ \\
\hline Antegrade flow & $\begin{array}{l}\text { Retrograde flow } \\
\text { (reflux) into } \\
\text { sinus }\end{array}$ & Antegrade flow & Retrograde flow & & Venous ectasia & $\begin{array}{l}\text { Associated w/ } \\
\text { progressive } \\
\text { myelopathy }\end{array}$ \\
\hline $\begin{array}{l}\text { No cortical } \\
\text { venous drain- } \\
\text { age/reflux }\end{array}$ & $\begin{array}{l}\text { No cortical ve- } \\
\text { nous drainage/ } \\
\text { reflux }\end{array}$ & & & & & \\
\hline
\end{tabular}

\section{Results}

\section{Study Population}

We retrospectively identified 111 patients who received endovascular treatment of an intracranial DAVF at our institution from January 2003 to June 2015. All CCFs, DAVFs primarily draining into the frontobasal/ethmoidal sinus or superior sagittal sinus, and lateral DAVFs of Cognard Types III-V (primarily involving cortical veins) were excluded, because in these cases our scientific issue (comparison of SP vs SO approach) was not relevant due to the anatomical preconditions. The remaining 36 patients had a DAVF of the lateral type with an initial grading of Cognard Types I-IIb prior to the intervention. These 36 patients were included in the analysis.

\section{Comparison Groups}

Of 36 patients with intracranial lateral DAVFs (Cognard Types I-IIb) treated by an endovascular approach during the evaluation period, 3 were treated by TVE $(8 \%)$, 12 received CSOE (33\%), 10 were treated by TAE $(28 \%)$, and 11 were treated using CSPE (31\%; Table 2).

In 2 cases, the therapeutic approach changed between 2 treatment sessions. In 1 case a TVE in the first intervention had to be extended to a CSOE in the second session; this patient therefore remained in the same comparison group (SO). In another case, treatment was initiated with CSPE and was extended to CSOE in the second treatment session, thus also leading to a paradigmatic shift into the other comparison group (SP to SO).

The final overall size $(n=36)$ of the comparison groups was thus 15 patients with SO embolization (42\%) versus

TABLE 2. Comparison groups

\begin{tabular}{cccccc}
\hline Group & $\begin{array}{c}\text { No. } \\
(\%)\end{array}$ & $\begin{array}{c}\text { Sex } \\
(\mathrm{M} / \mathrm{F})\end{array}$ & $\begin{array}{c}\text { Age in Yrs } \\
\text { (range) }\end{array}$ & $\begin{array}{c}\text { Time Period } \\
\text { (month/yr) }\end{array}$ & $\begin{array}{c}\text { Days From } \\
\text { Diagnosis to } \\
\text { (range) }\end{array}$ \\
\hline SO & $15(42)$ & $10: 5$ & $58(33-78)$ & & \\
\hline TVE & $3(8)$ & $2: 1$ & $54(49-57)$ & $2 / 06-7 / 08$ & $57(50-67)$ \\
\hline CSOE & $12(33)$ & $8: 4$ & $63(33-78)$ & $7 / 03-7 / 11$ & $87(0-413)$ \\
\hline SP & $21(58)$ & $12: 9$ & $55(22-77)$ & & \\
\hline TAE & $10(28)$ & $6: 4$ & $57(44-77)$ & $8 / 03-2 / 15$ & $174(0-1367)$ \\
\hline CSPE & $11(31)$ & $6: 5$ & $54(27-71)$ & $12 / 09-5 / 14$ & $45(0-141)$ \\
\hline
\end{tabular}

21 patients with SP embolization (58\%). Patient characteristics (sex and age), treatment periods, and time intervals from initial imaging to intervention of the different subgroups are summarized in Table 2.

\section{Fistula Grading Prior to Treatment}

Fistula grading in initial imaging prior to treatment included 11 Cognard Type I fistulas (31\%); 3 patients $(27 \%)$ received SO treatment, and 8 patients $(73 \%)$ were treated by SP embolization. Of 13 patients with Cognard Type IIa fistulas (36\%), $5(38 \%)$ received SO treatment while 8 $(62 \%)$ were treated by SP embolization. Of 6 patients with Cognard Type IIa+b fistulas (17\%), 4 (67\%) received SO treatment while in 2 cases $(33 \%)$ an SP treatment approach was chosen. Finally, the total collective included 6 patients with Cognard Type IIb fistulas (17\%), 3 of whom $(50 \%)$ received an SO and 3 an SP treatment approach (Table 4).

\section{Intervention Material}

In the SO comparison group $(n=15)$, material used included a combination of coils and Glubran in 3 patients (20\%), a combination of coils and Onyx in 5 patients (33\%), a combination of coils and Histoacryl in 5 patients (33\%), and a combination of coil, Glubran, and Histoacryl in 1 patient (7\%). One patient was treated by combining balloon, coils, Histoacryl, and Onyx (7\%, Table 3).

In the SP group $(n=21) 2$ patients were treated using only Histoacryl or Glubran, respectively (10\% each), 6 pa-

TABLE 3. Intervention materials used according to group

\begin{tabular}{cc}
\hline \multicolumn{1}{c}{ Materials Used } & No. $(\%)$ \\
\hline SO $(n=15)$ & $3(20)$ \\
\hline Coil, Glubran & $5(33)$ \\
\hline Coil, Histoacryl & $5(33)$ \\
\hline Coil, Onyx & $1(7)$ \\
\hline Coil, Glubran, Histoacryl & $1(7)$ \\
\hline Balloon, coils, Histoacryl, Onyx & \\
\hline SP $(n=21)$ & $2(10)$ \\
\hline Glubran & $2(10)$ \\
\hline Histoacryl & $6(29)$ \\
\hline Onyx & $11(52)$ \\
\hline Balloon, Onyx
\end{tabular}


TABLE 4. Primary success rate

\begin{tabular}{|c|c|c|c|c|c|}
\hline \multirow[b]{2}{*}{ Variable } & \multicolumn{5}{|c|}{ Cognard Classification (\%) } \\
\hline & $\begin{array}{c}\text { Definite } \\
\text { Occlusion* }\end{array}$ & I & Ila & $\| \mathrm{l}+\mathrm{b}$ & Ilb \\
\hline \multicolumn{6}{|l|}{ SO $(n=15)$} \\
\hline Prior to treatment & - & $3(20)$ & $5(33)$ & $4(27)$ & $3(20)$ \\
\hline After treatment & $14(93)$ & $1(7)$ & - & - & - \\
\hline \multicolumn{6}{|l|}{$S P(n=21)$} \\
\hline Prior to treatment & - & $8(38)$ & $8(38)$ & $2(10)$ & $3(14)$ \\
\hline After treatment & $15(71)$ & $4(19)$ & $2(10)$ & - & - \\
\hline
\end{tabular}

$-=$ none in class.

* In the SO group after treatment, there were 14 definite occlusions (93\%) and 1 Cognard type downgrade (7\%). In the SP group after treatment, there were 15 definite occlusions (71\%), 3 Cognard type downgrades (14\%), and 3 reductions of the arteriovenous shunt/fistula network (14\%). The data indicated a trend toward a higher degree of definitive therapy success in the SO group, but these differences were not statistically significant (Pearson chi-square $=$ $3.12, p=0.210$ ).

tients were treated by Onyx solely (29\%), and 11 patients were treated with a combination of Onyx and a compliant balloon (52\%; Figs. 1 and 2, Table 3).

\section{Complication Rate}

In the SO comparison group, 5 complications were reported among 15 treated patients (33\%). All complications occurred in the CSOE subgroup and consisted of a dissection of the internal carotid artery during the transarterial approach (7\%), a mediastinal perforation during the transvenous approach (7\%), intracranial hemorrhage during the first 24 hours after endovascular treatment (7\%), and a widespread cerebral venous thrombosis within the first 4 weeks after treatment (7\%). One patient suffered from bilateral thalamic infarction due to deterioration of a preexisting venous congestion after treatment, leading to death in the next few days after treatment (7\%; Table 5).

Two of 5 complications were related to the approach and thus not specific, whereas in 3 cases the complications were directly related to postinterventional changes in the venous drainage pattern and can thus be specifically assigned to the SO paradigm.

In the SP comparison group, no complication occurred in 21 treated patients. Differences in complication rates were calculated separately (overall SO vs SP: $p=0.008$, Fisher's exact test; SO specific, SO vs SP: $p=0.064$, Fisher's exact test; SO specific, CSOE vs CSPE: $p=0.217$, Fisher's exact test; Table 5).

\section{Fistula Grading After Treatment and Primary Success Rate}

In the SO group, 14 (93\%) of 15 patients were negative for a persistent arteriovenous shunt on the last imaging follow-up. One patient had a remaining Cognard Type I DAVF (7\%), but treatment had resulted in a downgrading on the Cognard scale (Table 4).

In the SP group, treatment led to a complete occlusion of the DAVF in $15(71 \%)$ of 21 cases. In 2 cases a Cognard Type II DAVF (10\%) and in 4 patients (19\%) a Cognard Type I DAVF remained. Downgrading on the Cognard scale could be achieved in 3 cases (14\%). In 3 cases (14\%) treatment did not result in a downgrading in the Cognard classification but showed a significant reduction of the arteriovenous shunt volume and/or the size of the fistula network. Treatment thus showed an improvement compared with the initial state in all of the cases (Table 4).

Our data thus indicate a trend toward a higher degree of definitive therapy success in the SO group; however, these differences were not statistically significant (Pearson chisquare $=3.12, p=0.210$; Table 4).

\section{Long-Term Follow-Up}

Questionnaire Response Rate and Duration

The response rate for the standardized questionnaires in the individual subgroups was as follows: $7(63 \%)$ of 11 patients in the CSPE subgroup, 7 (58\%) of 12 patients in
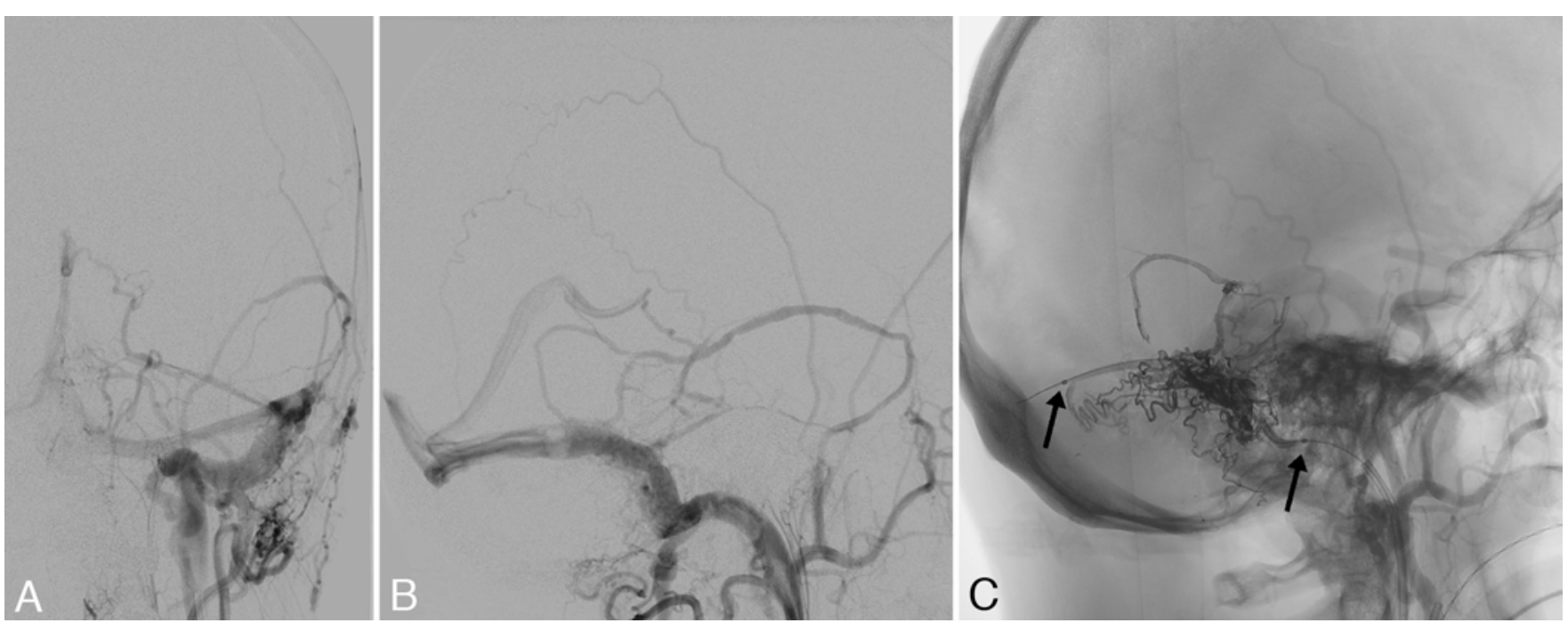

FIG. 1. Digital subtraction angiogram showing a Cognard Type lla+b lateral DAVF prior to CSPE. A and B: Left external carotid artery (ECA) frontal and lateral views. C: Lateral view of perisinusoideal Onyx cast after embolization, and a compliant balloon. (arrows) deflated. 


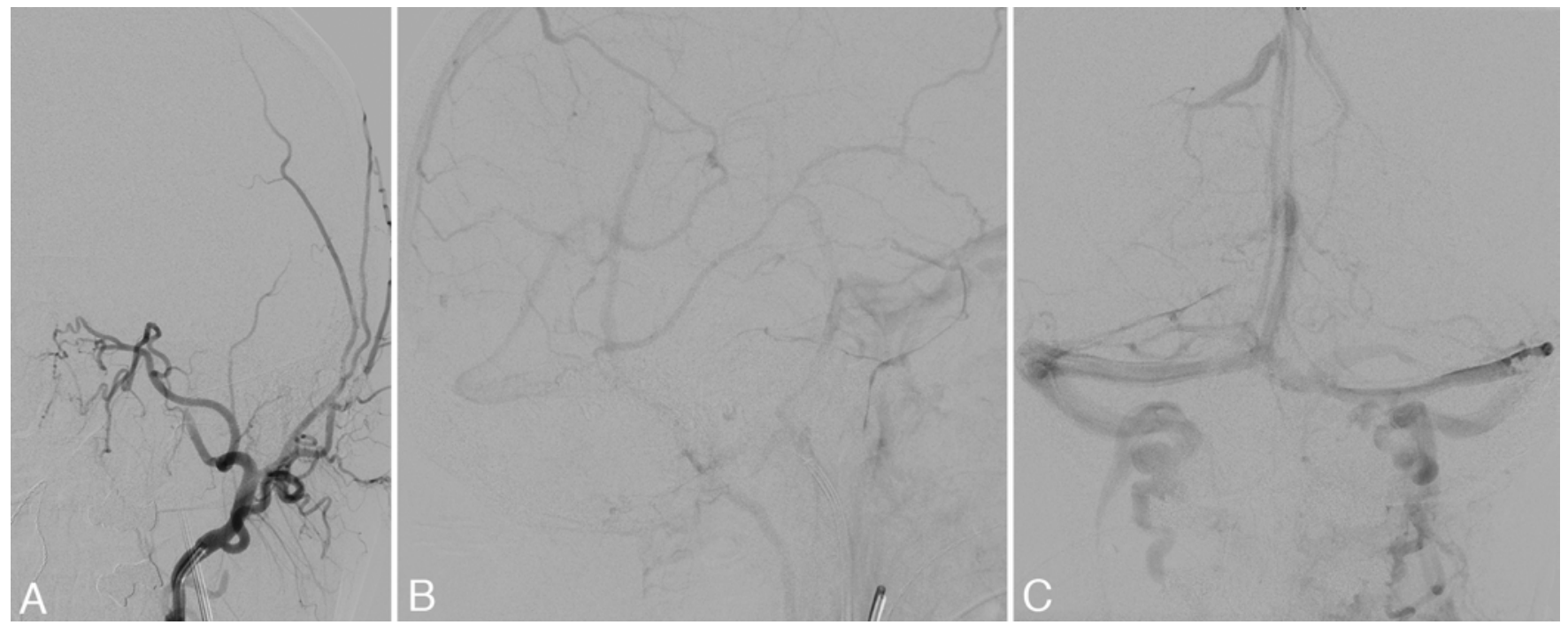

FIG. 2. Digital subtraction angiograms showing a Cognard Type lla+b lateral DAVF after CSPE. A: Left ECA frontal oblique view showing definitive occlusion. B: Left common carotid artery (CCA) lateral view demonstrating antegrade flow in the vein of Labbé. C: Left CCA frontal view showing antegrade flow in both the transverse and sigmoid sinus.

the CSOE subgroup, 7 (70\%) of 10 patients in the TAE subgroup, and $3(100 \%)$ of 3 patients in the TVE subgroup (Table 6).

The overall return rate of the standardized questionnaire in the comparison groups was 14 of 21 patients in the SP comparison group and 10 of 15 patients in the SO comparison group, thus $67 \%$ across both comparison groups (Table 6).

The duration of long-term follow-up, defined as the mean time interval between treatment and date indicated on the questionnaire in the individual subgroups, was as follows: 35 months (range 11-47 months) in the CSPE subgroup, 89 months (range 49-134 months) in the CSOE subgroup, 71 months (range 31-136 months) in the TAE subgroup, and 92 months (range 76-108 months) in the TVE subgroup (Table 6).

\section{Cognitive Deficits}

In the SO group 1 patient with a Cognard Type IIa+b DAVF draining into the left transverse sinus experienced a first acute severe epileptic state prior to treatment. MRI at admission showed venous congestion in the left temporal lobe. After successful CSOE (no arteriovenous shunt) with Histoacryl and coils, clinical symptoms persisted. Followup MRI showed progressive venous congestion without evidence of an inner cerebral vein thrombosis, which finally led to bithalamic infarction and the death of the patient during the next few days after embolization. In the SP comparison group, $1(8 \%)$ of 13 patients reported mild cognitive deficits not known prior to treatment. However, deterioration or even the presence of the cognitive deficits was never formally diagnosed, but based on the patient's subjective impression (Table 7).

TABLE 5. Complication rate*

\begin{tabular}{|c|c|c|c|c|c|}
\hline Group & $\begin{array}{c}\text { Complications } \\
(\%)\end{array}$ & Complication & $\begin{array}{c}\text { SO } \\
\text { Specific }\end{array}$ & $\begin{array}{l}\text { Cognard Type \& } \\
\text { Symptoms Prior to } \\
\text { Treatment }\end{array}$ & $\begin{array}{l}\text { Cognard Result } \\
\text { After Treatment }\end{array}$ \\
\hline$S O(n=15)$ & $5(33)$ & & & & \\
\hline CSOE & & $\begin{array}{l}\text { Dissection of the internal carotid } \\
\text { artery }\end{array}$ & No & & \\
\hline CSOE & & $\begin{array}{l}\text { Mediastinal perforation during trans- } \\
\text { venous approach }\end{array}$ & No & & \\
\hline CSOE & & $\begin{array}{l}\text { Venous congestion, bithalamic } \\
\text { infarction, persisting disturbance } \\
\text { of consciousness, death }\end{array}$ & Yes & Ilb, epileptic state & Definite occlusion \\
\hline CSOE & & $\begin{array}{l}\text { Secondary extended sinovenous } \\
\text { thrombosis } 4 \text { wks after treatment }\end{array}$ & Yes & Ila+b, chronic headache & Definite occlusion \\
\hline CSOE & & $\begin{array}{l}\text { ICB due to venous congestion } 24 \mathrm{hrs} \\
\text { after treatment }\end{array}$ & Yes & Ila, pulsatile tinnitus & $\begin{array}{l}\text { Definite occlusion after } \\
\text { SO re-intervention }\end{array}$ \\
\hline$S P(n=21)$ & 0 & - & - & - & - \\
\hline
\end{tabular}

* Results of group comparisons using Fisher's exact test: $p=0.008$ (overall SO vs SP), $p=0.064$ (SO specific, SO vs SP), and $p=0.217$ (SO specific, CSOE vs CSPE). 
TABLE 6. Long-term follow-up results

\begin{tabular}{cccc}
\hline & & \multicolumn{2}{c}{ Follow-Up (mos) } \\
\cline { 3 - 4 } Group & Return Rate $(\%)$ & Mean & Range \\
\hline SO & $10 / 15(66.66)$ & 90.40 & $49-134$ \\
\hline TVE & $3 / 3(100.0)$ & 91.67 & $76-108$ \\
\hline CSOE & $7 / 12(58.33)$ & 89.14 & $49-134$ \\
\hline SP & $14 / 21(66.66)$ & 53.34 & $11-136$ \\
\hline TAE & $7 / 10(70.0)$ & 71.29 & $31-136$ \\
\hline CSPE & $7 / 11(63.63)$ & 35.43 & $11-47$ \\
\hline
\end{tabular}

\section{Chronic Headache}

In the SO comparison group, 2 (22\%) of 9 patients suffered from chronic headache prior to treatment. Both patients recovered from their headache after treatment, whereas in another patient, chronic headache newly developed after treatment. In the SP comparison group, 1 (8\%) of 13 patients suffered from chronic headache prior to treatment, which completely disappeared after treatment (Table 7).

\section{Pulsatile Tinnitus}

All patients in both comparison groups suffered from a pulsatile tinnitus prior to treatment. At the time of our last follow-up interview, 2 of 13 patients in the SP comparison group and 2 of 9 patients in the SO comparison group had a residual bruit (Table 7).

\section{Vertigo}

In the SO comparison group, 2 (22\%) of 9 patients, and in the SP comparison group, 2 (15\%) of 13 patients suffered from vertigo prior to treatment. In the $\mathrm{SO}$ comparison group, all patients recovered from their vertigo, whereas 1 patient in the SP comparison group reported persistent vertigo after treatment (Table 7).

\section{Overall Tendency of Long-Term Follow-up}

The overall tendency of the long-term follow-up of the patients' subjective benefit from treatment was excellent. In the SO group, 9 of 10 patients observed a subjective improvement of their overall state of health compared with the state prior to treatment. In 1 of 10 patients the clinical state deteriorated either due to, or at least despite, SO treatment. In the SP group, all 13 patients reported an improvement in their health as a result of the treatment (Table 7).

\section{Discussion}

Since the invention of large-lumen compliant balloons and Onyx liquid embolic agent, therapy options for DAVF have expanded. The main advantage of this CSPE approach is the preservation of the natural venous drainage pattern by keeping the diseased sinus segment open through temporary balloon protection during the transarterial fistula embolization (Figs. 1 and 2). Sinus-preserving treatment variants are now performed in many centers; however, there has not yet been a structured evaluation of their benefit. In this article we thus aimed for a retrospective comparison of the different approaches of endovascular treatment for lateral DAVFs of low and intermediate grades (Cognard Types I-IIb).

Our analysis was restricted to this collective because only the anatomical conditions of these lateral DAVFs permit a combined approach with either occlusion or preservation of the diseased sinus segment. Whereas any treatment effort should come with the lowest possible complication rate, this principle is particularly relevant in the case of a low-grade lateral DAVF (Cognard Types I-IIa), which offers only a relative treatment indication. We hypothesized that any approach preserving the involved sinus segment offers a more favorable benefit-risk ratio-even regarding long-term follow-up-than an SO paradigm.

Sample size and baseline characteristics of our patients indicate a good comparability between the 2 collectives.

TABLE 7. Course of clinical symptoms before and after treatment

\begin{tabular}{|c|c|c|c|c|c|}
\hline \multirow[b]{2}{*}{ Symptom } & \multirow[b]{2}{*}{ Before Treatment (\%) } & \multirow[b]{2}{*}{ After Treatment (\%) } & \multicolumn{3}{|c|}{ Tendency } \\
\hline & & & Worse & Equal & Bette \\
\hline \multicolumn{6}{|c|}{ Cognitive deficits } \\
\hline SO $(n=10)$ & $1 / 10(10)$ & $1 / 10(10)$ & - & 1 & - \\
\hline$S P(n=13)$ & $0 / 13(0)$ & $1 / 13(7.69)$ & 1 & - & - \\
\hline \multicolumn{6}{|c|}{ Chronic headache } \\
\hline $\mathrm{SO}(n=9)$ & $2 / 9(22.22)$ & $1 / 9(11.11)$ & 1 & - & 2 \\
\hline$S P(n=13)$ & $1 / 13(7.69)$ & $0 / 13(0)$ & - & - & 1 \\
\hline \multicolumn{6}{|c|}{ Pulsatile tinnitus } \\
\hline $\mathrm{SO}(\mathrm{n}=9)$ & $9 / 9(100)$ & $2 / 9(22.22)$ & - & 2 & 7 \\
\hline$S P(n=13)$ & $13 / 13(100)$ & $2 / 13(15.38)$ & - & 2 & 11 \\
\hline \multicolumn{6}{|l|}{ Vertigo } \\
\hline $\mathrm{SO}(\mathrm{n}=9)$ & $2 / 9(22.22)$ & $0 / 9(0)$ & - & - & 2 \\
\hline$S P(n=13)$ & $2 / 13(15.38)$ & $1 / 13(7.69)$ & - & 1 & 1 \\
\hline \multicolumn{6}{|c|}{ Overall tendency } \\
\hline SO $(n=10)$ & & & $1 / 10$ & & $9 / 10$ \\
\hline$S P(n=13)$ & & & & & $13 / 13$ \\
\hline
\end{tabular}


There are small differences in the mean delay from initial imaging to treatment (109 days in the SP group vs 72 days in the SO group) and in the time period of treatment (August 2003-February 2015 in the SP group vs July 2003July 2011), but these differences are not likely to cause any bias between the 2 groups.

Fistula grading according to the Cognard classification prior to treatment differed slightly between the 2 groups. In the SP group, mostly lateral DAVFs of a fistula grade no worse than Cognard Type IIa were treated, whereas fistula grading was more or less evenly distributed between Cognard Types I-II in the SO group. This trend indicates that our interventional team took advantage of the opportunities offered by the newly developed CSPE approach to also treat lower-grade type fistulas in the past few years. However, we do not believe that this difference causes a relevant bias.

The rate of remaining DAVFs was higher in the SP group, in which $29 \%$ of the patients had a remaining arteriovenous shunt on the last DSA imaging follow-up compared with only 1 (7\%) of 15 patients in the SO group. Even if this difference in the primary success rate was not statistically significant $($ Pearson chi-square $=3.12, \mathrm{p}=$ 0.210 ), our data indicate that SO approaches are a more effective treatment option, offering a higher rate of definitive fistula occlusion compared with SP techniques (93\% SO vs $71 \%$ SP). However, in both collectives, no final therapeutic result worse than Cognard Type IIa was achieved. This means that the primary therapeutic goal in Cognard Types IIa $+b$ and IIb fistulas-the risk reduction for intracranial hemorrhage-was achieved in all cases.

In contrast, the complication rate was significantly higher in the SO group. While in the SP group no complication (0\%) occurred among 21 treated patients, 5 complications (33\%) were reported among 15 treated patients in the SO group, especially in the CSOE subgroup (overall comparison SO vs SP: $p=0.008$, Fisher's exact test). Even after removing the 2 complications not directly related to postinterventional changes of the intracranial hemodynamics from the calculation and comparing only the CSPE versus the CSOE subgroup, the difference remains a stable trend (SO specific, SO vs SP: $p=0.064$, Fisher's exact test; SO specific, CSOE vs CSPE: $p=0.217$, Fisher's exact test). The higher rate of definitive fistula occlusion in the SO comparison group was thus attained at the price of a significantly higher complication rate.

The return rate for our questionnaire of two-thirds of the total collective was high and equally distributed across both groups (67\% each). With the exception of 1 patient in the SO group who died during follow-up, all of the interviewed patients reported a subjective benefit for their state of health in both groups. Even if the missing values may cause a bias in the assessment of the long-term success rates, our data indicate that endovascular treatment of DAVFs, whatever the type of approach, is advantageous to the patient.

There are several limitations of our study that need to be mentioned. The small sample sizes and differences between groups, as well as the small event rate, make definitive conclusions impossible. Complication assignment was widely spread among the surgeons participating in endovascular fistula treatment in our department, but mathematically it was not completely equally distributed. Although we do not assume that this fact caused a relevant bias, we cannot formally exclude this possibility. Furthermore, some of our data were collected on the basis of a subjective questionnaire that was only returned by some of the patients. All these facts limit our findings. However, we believe that our results allow for our conclusions and recommendations.

To sum up, the repertoire of endovascular treatment for lateral DAVFs includes different options, of which SP techniques present with an excellent complication rate, whereas SO techniques offer a higher rate of definitive fistula occlusion that comes with the cost of severe complications in some cases. Choice of therapeutic paradigm therefore should be a direct consequence of the fistula grade prior to treatment. In all cases of relative treatment indication (Cognard Types I-IIa), an SP approach should be the primary choice. It offers a good primary success rate in combination with a very low complication rate. The patients are likely to benefit from treatment, even in long-term follow-up. An SP approach also should be preferred in the case of an initial fistula grade of Cognard Types IIa+b-IIb. Our data show that the primary therapeutic goal (prevention of intracranial hemorrhage by a downgrading to fistula grade no worse than Cognard Type IIa) was achieved in all cases. Even in cases with a persistent residual arteriovenous shunt, in the SP comparison group patients reported a subjective benefit from treatment in long-term follow-up.

Unlike on the arterial side in which a temporary vessel occlusion causes immediate clinical symptoms, there is no clear linear correlation between the occlusion of a sinus and its resulting clinical symptoms. This fact hampers a reliably safe, provocative testing procedure, predicting if a specific sinus segment is essential for the intracranial venous drainage. Currently, only careful analysis of the intracranial venous drainage pattern considering all acquired DSA series allows for an exploratory, yet not definite, risk assessment of an intended sinus occlusion. Our data show this practice is far from being reliably safe. We therefore recommend to renounce iatrogenic sinus occlusion whenever it is possible and to prefer an SP therapy option. The use of SO approaches should be restricted to cases in which SP treatment efforts fail to achieve a downgrading of the fistula to a grade no worse than Cognard Type IIa.

While 2 of 3 complications directly related to the sinus occlusion technique occurred in 2003 and 2006, respectively, thus at a time when the combination of Onyx and large-volume compliant balloons allowed for an SP treatment was not yet established, the case of 1 patient (initial fistula grade: Cognard Type IIa, 2010) clearly shows the risk of "overtreatment"; no definitive fistula occlusion by an SP approach could be achieved in the first treatment session. Despite an only relative treatment indication (subjectively disturbing pulsatile tinnitus), an SO approach was chosen in a second session, which led to secondary venous congestion and intracranial hemorrhage a few days later. As long as the primary therapeutic goal (remaining fistula grade no worse than Cognard Type IIa) can be achieved by an SP approach, we thus recommend leaving a small remaining fistula rather than risking severe secondary complications resulting from an iatrogenic sinus occlusion. 


\section{Conclusions}

Endovascular treatment is an effective treatment option for low- and intermediate-grade intracranial lateral DAVFs, offering an excellent rate of subjective benefit to the patient, even in long-term follow-up. For a few years now endovascular therapeutic options have included a sinus-preserving approach, combining large compliant balloons and Onyx liquid embolic agent. Despite some limitations such as small sample size and event rates, differences between groups, and subjective questionnaires, based on our data we recommend choosing an SP approach in all cases as the primary therapeutic option. As long as prevention of intracranial hemorrhage by a downgrading to fistula grade no worse than Cognard Type IIa can be achieved using an SP approach, we recommend leaving a small remaining fistula rather than risking severe secondary complications resulting from a sinus occlusion. The choice of an SO technique should be restricted to cases in which SP treatment efforts fail to achieve a downgrading of the underlying fistula to a grade no worse than Cognard Type IIa.

\section{References}

1. Borden JA, Wu JK, Shucart WA: A proposed classification for spinal and cranial dural arteriovenous fistulous malformations and implications for treatment. J Neurosurg 82:166179, 1995

2. Brown RD Jr, Flemming KD, Meyer FB, Cloft HJ, Pollock BE, Link ML: Natural history, evaluation, and management of intracranial vascular malformations. Mayo Clin Proc 80:269-281, 2005

3. Cognard C, Gobin YP, Pierot L, Bailly AL, Houdart E, Casasco A, et al: Cerebral dural arteriovenous fistulas: clinical and angiographic correlation with a revised classification of venous drainage. Radiology 194:671-680, 1995

4. Cognard C, Houdart E, Casasco A, Gabrillargues J, Chiras J, Merland JJ: Long-term changes in intracranial dural arteriovenous fistulae leading to worsening in the type of venous drainage. Neuroradiology 39:59-66, 1997

5. Cognard C, Januel AC, Silva NA Jr, Tall P: Endovascular treatment of intracranial dural arteriovenous fistulas with cortical venous drainage: new management using Onyx. AJNR Am J Neuroradiol 29:235-241, 2008

6. Davies MA, terBrugge K, Willinsky R, Coyne T, Saleh J, Wallace MC: The validity of classification for the clinical presentation of intracranial dural arteriovenous fistulas. J Neurosurg 85:830-837, 1996

7. Davies MA, terBrugge K, Willinsky R, Wallace MC: The natural history and management of intracranial dural arteriovenous fistulae. Part 2: aggressive lesions. Interv Neuroradiol 3:303-311, 1997

8. Halbach VV, Higashida RT, Hieshima GB, Wilson CB, Hardin CW, Kwan E: Treatment of dural fistulas involving the deep cerebral venous system. AJNR Am J Neuroradiol 10:393-399, 1989

9. Kwon BJ, Han MH, Kang HS, Chang KH: MR imaging findings of intracranial dural arteriovenous fistulas: relations with venous drainage patterns. AJNR Am J Neuroradiol 26:2500-2507, 2005

10. Mironov A: Cranial dural arteriovenous fistulas: clinical findings and radiologic diagnostics. Klin Neuroradiol 15:32-39, 2005

11. Nogueira RG, Dabus G, Rabinov JD, Eskey CJ, Ogilvy CS, Hirsch JA, et al: Preliminary experience with onyx embolization for the treatment of intracranial dural arteriovenous fistulas. AJNR Am J Neuroradiol 29:91-97, 2008

12. Roy D, Raymond J: The role of transvenous embolization in the treatment of intracranial dural arteriovenous fistulas. Neurosurgery 40:1133-1144, 1997

13. Satomi J, van Dijk JMC, terBrugge KG, Willinsky RA, Wallace MC: Benign cranial dural arteriovenous fistulas: outcome of conservative management based on the natural history of the lesion. J Neurosurg 97:767-770, 2002

14. Tomak PR, Cloft HJ, Kaga A, Cawley CM, Dion J, Barrow DL: Evolution of the management of tentorial dural arteriovenous malformations. Neurosurgery 52:750-762, 2003

15. van Dijk JMC, terBrugge KG, Willinsky RA, Wallace MC: Clinical course of cranial dural arteriovenous fistulas with long-term persistent cortical venous reflux. Stroke 33:12331236,2002

16. Zipfel GJ, Shah MN, Refai D, Dacey RG Jr, Derdeyn CP: Cranial dural arteriovenous fistulas: modification of angiographic classification scales based on new natural history data. Neurosurg Focus 26(5):E14, 2009

\section{Disclosures}

The authors report no conflict of interest concerning the materials or methods used in this study or the findings specified in this paper.

\section{Author Contributions}

Conception and design: Ertl, Brückmann, Crispin, Fesl.

Acquisition of data: Ertl, Kunz, Fesl. Analysis and interpretation of data: all authors. Drafting the article: Ertl, Brückmann, Kunz, Fesl. Critically revising the article: all authors. Reviewed submitted version of manuscript: all authors. Approved the final version of the manuscript on behalf of all authors: Ertl. Statistical analysis: Ertl, Crispin. Administrative/technical/material support: Ertl, Brückmann, Kunz, Crispin. Study supervision: Ertl, Brückmann, Fes1.

\section{Correspondence}

Lorenz Ertl, Department of Neuroradiology, University of Munich, Grosshadern Campus, Marchioninistr. 15, Munich D-81377, Germany.email: lorenz.ert1@med.uni-muenchen.de. 